\title{
Literacy Advantages Beyond Reading: Prediction of Spoken Language
}

\author{
Falk Huettig ${ }^{1,2, \star}$ and Martin J. Pickering ${ }^{3}$
}

Literacy has many obvious benefits: it exposes the reader to a wealth of new information and enhances syntactic knowledge. However, we argue that literacy has an additional, often overlooked, benefit: it enhances people's ability to predict spoken language thereby aiding comprehension. Readers are under pressure to process information more quickly than listeners and reading provides excellent conditions - in particular, a stable environment - for training the predictive system. It also leads to increased awareness of words as linguistic units and to more fine-grained phonological and additional orthographic representations, which sharpen lexical representations and facilitate the retrieval of predicted representations. Thus, reading trains core processes and representations involved in language prediction that are common to both reading and listening.

\section{Literacy and Prediction}

Learning to read during childhood or adulthood transforms people's lives. It gives readers access to a wealth of new information; for instance, through novels, factual articles, and public notices [1]. 'Book language' is also different from conversational speech because the more sophisticated language of written text involves more complex grammar than typical everyday speech interactions [2]. For this reason, exposure to written language enhances syntactic knowledge. The frequency of shared reading with parents at 24 months, for instance, predicts children's comprehension of syntactically complex sentences at 30 months [3]. Literacy (see Glossary) improves memory [4], visual search [5], and mirror image discrimination abilities [6] and changes cortical $[7,8]$ and subcortical [9] brain networks. However, we argue here that literacy has an important additional benefit: it causes people to predict spoken language and thereby aids understanding. People predict extensively during both spoken (e.g., [10,11]) and written (e.g., [12,13]) language comprehension, and such prediction facilitates comprehension (e.g., $[14,15])$. We do not suggest that people always predict (but see $[16,17]$ ) but they may predict aspects of language such as meaning or grammar (e.g., a noun referring to something edible) even if the word itself is unpredictable. They pre-activate predictable upcoming information and use it to reduce processing load if the upcoming information is subsequently encountered. In other words, they can focus on checking for matches between it and the input.

In this Opinion article, we argue that literacy enhances prediction and that this ability to predict transfers to the comprehension of spoken language. Literacy enhances background knowledge and thus in part reflects secondary influences of reading acquisition. We define secondary influences as indirect consequences of book language; that is, those influences on prediction that can also be acquired by exposure to audio books (e.g., learning the more elaborate language and complex grammar and extensive vocabulary of written text). However, literacy also has primary influences on prediction because people develop predictive skills through reading and these skills transfer from reading to language processing as a whole. We define primary influences of reading as those that are directly linked to the physical act of reading (e.g., the timing and regularity of

\section{Highlights}

Proficient readers predict spoken language faster than less-proficient readers and illiterate adults. This reflects in part secondary correlates of reading acquisition, such as having larger vocabulary knowledge and working memory capacity.

However, we also argue that there are primary influences of reading behavior on speech prediction. We discuss several properties of the reading environment that support prediction and transfer from reading to spoken language processing.

Reading acquisition boosts prediction of upcoming speech because it trains shared processes (e.g., formation of predictive dependencies) and sharpens shared representations (e.g., lexical, syntactic).

\section{${ }^{1}$ Max Planck Institute for} Psycholinguistics, Nijmegen, The Netherlands

${ }^{2}$ Centre for Language Studies, Radboud University, Nijmegen, The Netherlands ${ }^{3}$ University of Edinburgh, Edinburgh, UK

${ }^{*}$ Correspondence:

falk.huettig@mpi.nl (F. Huettig). 
saccadic eye movements during reading, exposure to printed word forms, word bias created by encountering word separation in written language). The assessment of benefits of literacy beyond reading is an important and timely issue. It has been suggested that rather than teaching illiterates in developing countries how to read (or functional illiterates across the world how to read better and more), artificial intelligence voice recognition and voice assistants provide an answer to dealing with low literacy [18]. The arguments outlined below suggest that such a strategy might be counterproductive and have some unintended, far-reaching consequences.

\section{Literacy Influences Prediction in Spoken Language Processing}

Several studies have shown the effects of literacy on prediction in spoken language processing. Eye-tracking studies have shown that adults who are proficient readers predict objects in their surroundings whose names are predictable following a sentence context but low-literate adults do not [19]. Similarly, children who are good readers were better at predicting the reference of concurrent speech in their visual environment than less-proficient readers and word-reading scores were a robust predictor of anticipatory looks [20]. These studies involved different types of participant populations (low and highly proficient adult readers, low and highly proficient developing readers), different types of spoken language materials (adjective-particle-noun constructions, thematic arguments consistent with a verb's selectional restrictions), and different languages (Hindi, German), thereby providing converging evidence for the effects of literacy on speech prediction. Even college students in the USA with higher literacy (as assessed in author recognition, vocabulary knowledge, word reading, reading habits, and weekly reading time) predict more upcoming spoken language than US college students with lower literacy [21]. Moreover, a recent electrophysiological study has shown that higher reading proficiency in adults is associated with reduced negativity over anterior channels as early as 170-300 ms after target onset when listening to a strongly predictable spoken target word compared with adults with lower reading proficiency [22]. It is important to stress here that this does not mean that without reading experience listeners make no predictions. Two-year-olds make some predictions about up-coming spoken language [11,23] (although 4-5-year-olds do not seem to predict the form of upcoming words [24]). However, literate adults become better and often extremely skilled predictors.

\section{Why Does Prediction in Reading Enhance Prediction in Spoken Language?}

It is important at this juncture to note that there has been some controversy about the robustness of transfer effects in the cognitive sciences, but it mainly relates to the replicability of so-called far transfer effects across domains [25]; for example, whether working memory training improves other distinct mental abilities such as fluid intelligence and attention [26,27]. However, there is little doubt about near transfer effects; that is, that training can affect subsequent performance in related abilities that did not receive additional training [28]. For example, working memory training improves reading because successful reading comprehension relies on working memory processes such as connecting different parts of a text and maintaining content-relevant information for the construction of a situation model [29-31].

We suggest that there are two explanations for near transfer from literacy to spoken-language prediction. First, exposure or training within a particular domain affects core processes of the system that are shared. Some of the processes that are shared during reading and listening result in the formation of predictive dependencies; for example, associations [32] or error-based learning of dependencies among representations [33,34]. Second, reading experience transfers to speech because it exerts its effect on at least some identical representations. It is widely accepted that written and spoken language processing involve many shared representations (e.g., lexical [35,36], syntactic [37]) and those that are not shared (e.g., orthographic and phonetic/phonological representations) can directly impact each other (e.g., speech processing is

\section{Glossary}

Associations: a mental connection between two mental concepts, states, or events due to experience.

Compound words: two (originally independent) words used together to yield a new meaning (e.g., daydream, however, nobody).

Dyslexia: a reading disorder in which affected individuals show impaired reading abilities despite normal levels of intelligence.

Electrophysiological studies: experimental studies that monitor the electrical activity of the brain over a period of time.

Error-based learning: an instance of learning by which the system that supports learning readjusts itself when encountering an error to improve performance at the next encounter. Far transfer effects: training in a particular domain that impacts (typically improves) abilities across domains (e.g., music training increasing general intelligence).

Fixations: maintenance of eye gaze on a single location.

Functional illiterates: individuals who show inadequate reading abilities despite having received some reading instruction.

Grapheme: smallest unit of a writing system of a language.

Literacy: knowledge and competence in reading and writing (although it can be extended to other areas).

Magnetoencephalography: a

neuroimaging technique of measuring brain activity by recording magnetic fields produced by electric currents in the brain. Morpheme: smallest grammatical unit in a language.

Near transfer effects: training in a particular domain that impacts (typically improves) domain-specific abilities (e.g., working memory training improving performance in tasks that partly rely on working memory).

Phoneme: a unit of sound that distinguishes spoken words in a language (e.g., /p/ in pat vs /b/ in bat). Prediction: pre-activation of upcoming information.

Saccades: rapid eye movements with large amplitude used to change the direction of gaze.

Saccadic eye movements: eye movements that involve quick jumps in eye gaze (saccades) between two or more periods of relative stability (fixations). 
affected by orthography [38]). We suggest that both secondary and primary influences of reading acquisition on prediction affect the core processes and representations.

\begin{abstract}
Secondary Influences of Reading That Affect Predictive Comprehension
Vocabulary Knowledge

Preliterate children and illiterate adults learn words mostly from oral social interactions [39]. Eventually, however, oral contexts cease to help vocabulary development because everyday conversations rarely contain words beyond the most frequent ones [40]. Reading experience greatly improves vocabulary knowledge because the language of written texts exposes language users to new words. In addition, experimental evidence shows that more frequent encounters with words increase the depth of word understanding $[41,42]$. Less skilled readers are less able to use context to derive the meaning of words [43]. There are dramatic differences in the number of words read per year, with the most avid child readers (98th-percentile rank) encountering over four million words a year and children who rarely read (10th-percentile rank) encountering only about 50000 words [44]. Importantly, there is evidence that reading habits contribute to vocabulary growth over and above general cognitive ability [45]. Thus, reading has specific and direct effects on vocabulary growth.
\end{abstract}

Secondary influences of reading such as increased vocabulary knowledge affect both processes and representations shared with spoken language. Greater and deeper vocabulary knowledge acquired from written sources, for instance, leads to more sophisticated interconnected networks of words (e.g., stronger associations among representations $[46,47])$. Increased depth of word understanding, moreover, results in greater lexical precision, which facilitates the activation of target lexical representations and minimizes the activation of competing alternatives [48,49]. Such developments also benefit spoken language because most predictive (e.g., associative) dependencies and linguistic (e.g., semantic) representations are shared between written and spoken language [35,36].

Importantly, studies with children $[11,50,51]$ and adults $[50,52,53]$ suggest that increased vocabulary knowledge enables increased prediction of spoken language. Crucially, studies have found that vocabulary knowledge robustly accounted for the unique variance of prediction of spoken language beyond production fluency and nonverbal IQ (Raven's progressive matrices) [52,53].

Verbal Working Memory

Reading letters, newspapers, magazines, or novels requires the integration of information over several sentences, paragraphs, or often even pages, and it requires keeping track of multiple entities and so enhances memory. Furthermore, the intensive practice of recoding skills during reading acquisition supports the development of short-term memory functions, including subvocal rehearsal and, as a consequence, working memory for serial order information $[4,54]$. Working memory according to influential models (e.g., $[55,56])$ is the ability to activate long-term memory representations and keep them active for online processing. An important feature of these models is that working memory capacity is mediated by an individual's expertise or experience and does not just reflect the processing resources available for any task at hand but is specifically operationalized as memory for verbal material. Both reading and listening require verbal working memory, which is shared for written and spoken language [4]. Spoken language prediction relies on this common verbal working memory [57].

Verbal working memory capacity in children increases as a function of reading rate. A study with 8-, 10-, and 12-year-old children, for example, concluded that the developmental increase in working memory span is attributable fully to the increase in mean reading rate [58]. Deficits in verbal working memory in individuals with reading disorders are well documented [59-61]. The notion that reduced and suboptimal reading experience at least partly explains the impairment in verbal working memory of individuals with reading disorders [62] is supported by many studies that have observed reduced verbal (but not spatial) working memory abilities in illiterate and 
low-literate people (e.g., [54,63]). Importantly, regression analyses show that verbal working memory contributes significantly more than nonverbal intelligence (as measured by performance in Raven's progressive matrices) to unique variance in speech prediction [57].

To conclude, substantial evidence links secondary influences of reading acquisition such as vocabulary knowledge and verbal working memory to enhanced spoken language prediction. The studies that also measured a general ' $g$-factor' show that general intelligence does not account for the influence of vocabulary knowledge and verbal working memory on spoken language prediction $[52,53,57]$. We now turn to the primary influences of reading on spoken language prediction.

\section{Primary Influences of Reading That Affect Predictive Comprehension}

A main reason for the differences in the tendency to predict between reading and listening relates to the speed of language processing. This is because of the different information processing bottlenecks in the two modalities. The bottleneck for processing spoken language is the speed of language production. Normal speech rates are 80-180 words per min. However, people can understand speech that is compressed to around twice this rate [64], so comprehension of normal speech, under good listening conditions at least, is well within comprehenders' abilities and bottom-up processing of the input typically suffices.

Reading, by contrast, is 'self-paced'. Thus, when learning to read there is an incentive to learn to predict. Skilled readers tend to read texts much faster than they process spoken language: they read English prose at approximately 250-300 words per min [65]. They (typically) have the goal of reading as fast as possible, primarily so that they can understand or act on what they encounter efficiently but also perhaps because they have a general tendency to maximize the rate of information processing. Indeed, reading is pretty much as fast as it can be (given limits on saccadic eye movements and speed of lexical access) and any attempt to read faster ('speed reading') impairs comprehension [66]. Readers do whatever they can to maximize and maintain this speed and therefore make use of predictive mechanisms [both in relation to upcoming words (i.e., general language predictions) and in relation to the specific written form (i.e., its shape and location)] whenever they can if they give them even a small advantage.

Experimental evidence for the importance of prediction in reading comes, for instance, from electrophysiological studies that show that a strongly constraining context is needed to facilitate word processing in lower-proficiency readers [67]. Moreover, the frontal positivity linked to predictive processing emerges only when reading rates are relatively fast [68]. Individuals who predict less in reading incur substantial processing costs for revising predictions [67].

\section{Properties of the Reading Environment That Affect Predictive Comprehension: Processes}

As a consequence of spending much of their lives comprehending regularly structured stimuli as fast as they can, literate people hone their ability to predict language. Prediction in reading is the result of learned relationships: between a word or broader context and a likely upcoming word or between visual forms (i.e., orthography or word shape). So, if I have repeatedly encountered 'book' after 'read the', and I then encounter 'read the', I can predict 'book' - the lexical item 'book', the sequence of letters, and the actual form on the page (e.g., its font and point size). Similar relationships hold of course for spoken language, but reading is special in that it supports the formation of predictive relations in several ways.

The Timing and Regularity of Eye Movements during Reading

Eye movements during reading involve a highly regular pattern of fixations and saccades, with readers learning to optimize their eye movements based on very few parameters (essentially, 
fixation duration and location [69]). Their ability to process information (how much is taken in during a fixation) is a consequence of this behavior and of the physiological limits of the eye. The timing of skilled readers' eye movements comes about as a result of satisfying the goals of reading as fast as possible while maintaining a good level of comprehension [70]. Through years of practice, skilled readers determine an optimal time to start programing a saccadic eye movement, to avoid remaining fixated on an already identified word for too long while also not leaving a word prematurely. Simulations suggest that readers tune word identification and eye movement systems in such a way that saccades from one word to the next are initiated before the fixated word is completely identified but which takes account of how much longer the word will take to identify $[71,72]$, thus ensuring that fixation time is optimized. In other words, readers use a number of cues to predict how quickly a word will be identified and thus determine the optimal time to begin programming a saccade. We suggest that these dynamics of eye movement behavior during reading, in particular the decision to initiate a saccade to the next word before the fixated word is fully identified, encourage readers to predict how long that word might take to identify (Box 1). The developing reader, of course, gets regular feedback about the success or otherwise of such predictions (e.g., have I moved my eye too early or too late relative to word identification?) and can learn successful predictive behavior (e.g., determining how early to begin programming a saccade). Readers thus learn the predictive dependencies between written input and, crucially (because lexical $[35,36]$ and syntactic [37] representations between written and spoken language are shared), predictive dependencies are also increased for the prediction of spoken language as a function of reading proficiency.

\section{Extreme Form-Invariance of Printed Forms}

Not only is reading behavior regular, but printed texts are regular too. Almost all tokens of an individual word within a text are practically identical (besides capitalization of the first letter of a sentence in written English). Intentional exceptions such as italics, cross-line hyphenations, changes in font size, and whole-word capitalization are rare, as are unintentional exceptions such as misspellings, typos, and smudges (or computer glitches) in most proof-read texts (although word spacing is a partial exception). Even differences between texts (related to font)

Box 1. The Timing of Saccadic Eye Movements during Reading

Readers of English and related languages/scripts take 175-200 ms to program and execute a saccadic eye movement [96]. Readers on average fixate a word for 200-250 ms before shifting eye gaze to the next word, with fixation durations being strongly influenced by a word's lexical characteristics. The beginning of programming the saccade to the next word thus typically occurs early during the processing of the currently fixated word. Word frequency affects the timing of the decision to initiate saccade programming $[97,98]$, implying that readers are in some way able to predict the difficulty of processing a word on the basis of relatively little 'bottom-up' information. Importantly, word frequency effects on saccade programming are unlikely to be explained by orthographic form familiarity effects independent of lexical access [99].

The regularity of saccadic eye movements means that a predictive strategy for when to begin programming a saccade can be learned and optimized - all that is necessary is to determine when the saccade should be programmed and where it should be directed. The question of when to program the saccade is captured by models such as E-Z Reader [69], which assume that the decision must occur early, specifically before word identification is complete (on completion of the socalled L1 phase). The precise process by which this occurs is unclear; Schotter [100] pointed out that such saccade planning is based on partial word recognition and is supported by partial information extracted from context [101]. However, importantly, we can be confident that readers engage in some form of prediction to facilitate eye movements in reading. Recent evidence also suggests a role for prediction in how readers target their saccades to a specific location within a word [102]. According to this work, readers predict how long a saccade from the word to the right of fixation to the word beyond this would need to be, to land in the center of the second word prior to either word being directly fixated. On fixation of the first word, this prediction is updated with information about the distance between the current fixation location and the center of the following word. The early prediction of the saccade length may accelerate how quickly readers are able to move between words. 
are systematic and regular (Figure 1A, Key Figure). Hence written form is highly predictable. If readers can predict the upcoming word, they can also predict the form of the word - exactly what shapes to expect on the next few centimeters of the page or screen. Moreover, the form of the prior context is also entirely regular (e.g., the form and location of 'The boy is flying his' is regular, just as much as 'kite'). Such regularity allows readers to set up consistent predictive models (a particular written context form predicts a particular written target form). There is good experimental evidence for such prediction of visual form (Box 2).

\section{Key Figure}

Properties of the Reading Environment That Affect Prediction

(A)

Extreme form-invariance of printed words Extreme form-invariance of printed words Extreme form-invariance of printed words Extreme form-invariance of printed words Extreme form-invariance of printed words Extreme form-invariance of printed words Extreme form-invariance of printed words

(B)

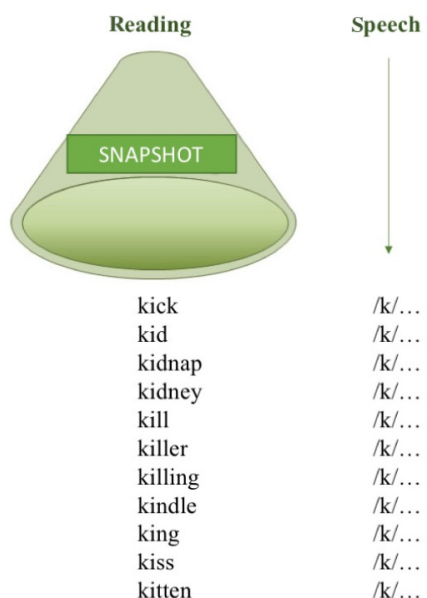

(C)

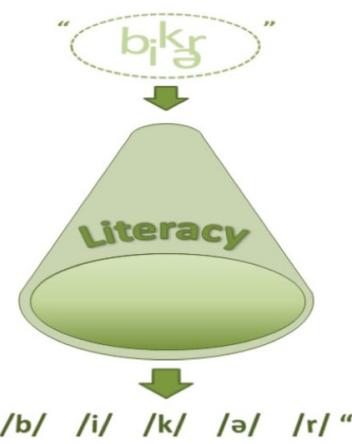

(D)

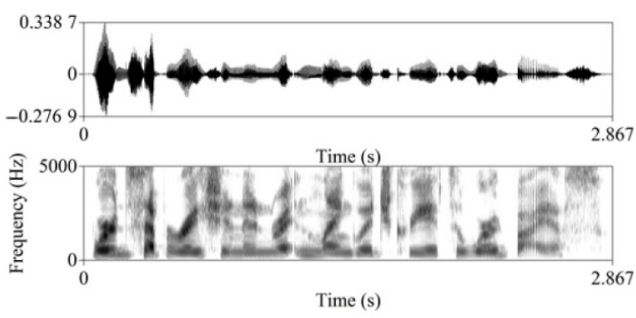

Word separation in written language creates a word bias.

Figure 1. (A) Printed words are extremely form-invariant, as shown by seven of the most-used print fonts (Arial, Times, Garamond, Calibri, Bodoni MT, Courier New, Gill Sans MT). By contrast, more than $60 \%$ of words in speech corpora deviate from their citation form [117]. Written form is therefore much more predictable than spoken form. This extreme regularity allows readers to set up consistent predictive models, as particular written context forms predict particular written target forms. (B) Parallel processing of multiple letters when reading means there is a clear one-step mapping between a 'snapshot' form and its associated representation (e.g., meaning) and thus the process of learning predictive relationships occurs in a regular manner at a specific time. (C) Learning to read leads to increased awareness of the compositional nature of speech and makes words more salient as a unit. (D) The sentence 'Word separation in written language creates a word bias' represented as a speech wave form and spectrogram and as printed sentence. The speech wave form and spectrogram reveal no clear 'pauses' between words and there are sometimes 'pauses' within the spoken words. Word separation in written language creates a stronger word bias than in continuous speech streams, making prediction during language processing much more viable. By sharp contrast, casual speech to which listeners are exposed in everyday life contains a huge amount of phonological reduction [118]. Casual speech is also full of disfluencies, as well as incomplete utterances and speech errors, which lead to low consistency [118]. Reductions are thus another source of variability that does not occur in written language (e.g., we do not change the printed letter 'e' to a schwa symbol). The presence of reduced forms in weakly constraining contexts increases the likelihood that word recognition will fail rather than that listeners compensate by trying to predict [119]. 
Box 2. Visual Form Is Predicted

The reader predicts a specific word form (including its size and font, not merely an orthographic representation) based on the context or uses such a form to predict a meaning. If the reader is incorrect, the reader can use error-based learning to adjust subsequent predictions. If correct, the prediction is strengthened. Over time, prediction during reading becomes very precise as a consequence of its form-invariance, and because some representations between written and spoken language are shared, predictive dependencies are also increased for the prediction of spoken language.

In a key study [103], participants were presented with written context that biased towards the prediction of a syntactic category (noun or verb participle) and a target noun with visual form features that were typical (e.g., soda) or atypical (e.g., infant) of nouns. Enhanced activity in the visual cortex using magnetoencephalography (an M100) was observed for the difference between the typical noun in the verb-biasing context and the typical noun in the noun-biasing context but not for atypical nouns (thus, the effect occurred only when there was a mismatch between the syntactic category and the visual form of the target word). An M100 effect is considered to be too early to be influenced by lexical access (syntactic category information in this case). This suggests that participants predicted the upcoming word's syntactic category and its visual form. The finding that the effect was localized to the visual cortex further implicates visual form prediction.

Parallel Processing of Multiple Letters in Written Word Recognition

Written input allows much more parallel processing than speech input. Parallel processing of multiple letters when reading means that readers get almost immediately a 'snapshot' of the morpheme (in long words), the whole word form, or even the whole form of compound words (Figure 1B). Thus, there is a clear (one-step) mapping between a 'snapshot' form and its associated representation (e.g., meaning), and so the process of learning the predictive relationship occurs in a regular manner at a specific time. The temporal unfolding of spoken word recognition is very different from the temporal dynamics of reading. In spoken word recognition the uniqueness point - the point at which only one remaining spoken word is compatible with the input - has an important influence on spoken word recognition [73]. By contrast, there is no evidence for a uniqueness point in written word recognition. A consequence of this is that spoken language is more geared towards prediction within a word (e.g., on the basis of a cohort; cf. [74]), while written language places more emphasis on prediction of relations between words (see Outstanding Questions).

There is strong experimental evidence for parallel processing of large units in written word recognition $[75,76]$. Readers process two-character compounds in Chinese using a 'whole-word' route [77], but even readers of alphabetic writing systems typically engage in morphemic or whole-word reading rather than sequential grapheme-phoneme conversion [75]. (This is the case for skilled readers and contrasts with the slow left-to-right grapheme-to-phoneme conversion strategy of the beginning reader [78]). In conclusion, proficient readers typically process morphemes or even the whole word form at once and therefore learn to use these large units as the basis for prediction. Importantly, processes such as associative learning and/or error-based learning are shared between reading text and listening to speech and result in the forming of predictive dependencies between shared (lexical and syntactic) representations that also enhance spoken language prediction.

\section{Properties of the Reading Environment That Affect Predictive Comprehension: Representations}

Increased Awareness of the Compositional Nature of Speech Units

To be able to read, people must map graphemes onto the corresponding sound units of spoken language. Proficient reading thus requires the knowledge that speech can be decomposed into smaller segments (Figure 1C). Without awareness that words in alphabetic scripts can also be decomposed into graphemes, readers cannot efficiently perform this mapping. Early forms of phonological knowledge and segmental awareness such as syllable onset and rhyme awareness can develop without any explicit teaching before reading instruction [79] but (preliterate) children and (iliterate) adults do not acquire more fine-grained segmental awareness unless they learn to read [80]. Phonemic 
awareness is something children and adult illiterates have to learn in the early stages of reading acquisition, and it develops from larger to smaller speech units [81,82]. This 'reading-induced' increased awareness of the compositional nature of speech makes words more salient as a whole (i.e., it creates a word bias) also during the processing of spoken language [82]. We discuss the relevance of this after the next (related) point concerning word separation in written language.

\section{Word Separation in Written Language}

A consequence of morphemic and whole word-form processing and word separation in written language (Box 3 ) is that the input in written language creates a word bias that is not the same as for spoken language processing (Figure 1D) [83]. The concept of a 'word' is an invention of the literate mind and a notion that is extremely difficult to grasp for illiterate people [82]. Illiterates are unable to divide utterances into words [82]. They also tend to produce whole sentences when asked to produce one long word [82,84]. Preliterate children and illiterate adults also repeat the whole sentence when asked to repeat only the last word of a spoken sentence [85]. Thus, it is well established that word separation in written language creates a stronger word bias than in continuous speech streams leading to more precise word representations. Enhanced lexical quality (i.e., sharpened representations) results in increased accuracy and fluency of word identification, retrieval of word meaning, the ability to learn new words, and the integration of words within discourse representations (see [49] for a review), thus making prediction during language processing much more viable, both when reading and when listening.

More Fine-Grained (Phonological) and Additional (Orthographic) Representations

Reading acquisition creates new neuronal connections between phonological and orthographic representations $[86,87]$. There is also evidence for lexical restructuring of phonological representations as a function of learning to read [88-90]. Recent behavioral and neuroimaging studies suggest that reading can affect speech processing via both activation of (additional) orthographic knowledge and more fine-grained phonetic/phonological representations [8]. Illiterates also have been observed to be less precise in phoneme discrimination tasks than literates [82]. Representations that are more precise reduce ambiguity and permit prediction that is more precise. Relatedly, there is experimental evidence that reading acquisition directly leads to faster retrieval of phonological representations [91]. Faster retrieval of representations permits faster prediction.

\section{Concluding Remarks}

Learning to read encourages people to learn to predict. 'Book language' has secondary effects on prediction in spoken language because it increases vocabulary knowledge and working memory capacity. Reading also has primary influences on speech prediction. It provides excellent conditions, in particular a stable environment, for training the predictive system. The regularity of eye-movement behavior in conjunction with the extreme regularity and form-invariance of printed

\section{Outstanding Questions}

Are listeners particularly good at predictions involving those words that they have encountered during reading (in which case the predictions will be more specific in nature; e.g., learning the printed form 'considerable' to predict the meaning CONSIDERABLE might generalize that knowledge to prediction about the spoken form 'considerable').

Is spoken language (comparatively) more geared towards the prediction of relations within a word than the prediction of relations between words?

Fully justified text has irregular interword space. Does reading fully justified text with variable word spacing impair predictive learning?

Changing font style improves the success of proof-reading. Font style influences distraction when reading under conditions of environmental noise. Does changing fonts (mid-text or between texts) make prediction more challenging because of the reduced regularity of the written symbols that support the precision of prediction?

Are people worse at predicting handwriting because of its greater variation in symbol forms?

Social media appear to encourage more variation (e.g., 'sloppy' spelling) than traditional print media. Do increased social media reading habits result in less predictive leaming?

\section{Box 3. Word Separation in Written Language}

Words in most (although not all; e.g., Chinese, Thai) of the world's modern writing systems are separated by empty space. Although some ancient texts were separated by either space or interpuncts, most Greek and Roman texts were written continuously without any punctuation or intratextual space (R.W. Müller, Inaugural dissertation, University of Tübingen, 1964). This made reading very challenging and led to a habit of oral reading and delegation of reading and writing to skilled slaves who served as professional readers. The modern habit of word separation in written language only became standard practice in Renaissance Italy and France and encouraged silent reading at a much faster pace [104]. Modern readers consider text without space (or punctuation) extremely difficult. Unspaced text slows reading and encourages oral reading and/or subvocal processing [105]. It also leads to a greatly reduced perceptual span of peripheral vision [106] and many more regressive eye movements [107] compared with spaced text. Most importantly, word separation creates a strong word bias when parsing text. Experimental evidence suggests that word spacing facilitates word recognition even in writing systems that do not standardly use them such as Thai [108] and Chinese [109]. 
forms and the parallel processing of multiple letters in written text means that prediction can be precisely honed. Reading leads to increased awareness of the compositional nature of speech units, word separation in written language, and more fine-grained phonological and additional orthographic representations, which sharpen lexical representations and facilitate predicted representations to be retrieved. Thus, reading trains core processes and representations involved in language prediction that are common to both reading and listening.

What kind of research and experimental evidence should be considered crucial to further test the account we have put forward here (see Outstanding Questions)? It is important that experimental studies more precisely quantify how much people actually read and that such studies assess rigorously the quality and type of people's reading experience (including home literacy environments and social media reading experience [92-94]). Correlational evidence from cross-sectional studies will remain useful but it is important to recognize their limitations with regard to confirming causal explanations. Computational modelling can play a role in testing mechanisms and representations explicitly. Results and hypotheses derived from cross-sectional studies and computational modelling must be confirmed by longitudinal intervention studies. The gold standard, we suggest, is tightly controlled, large-scale longitudinal studies with both developing and mature readers (Box 4) that meticulously monitor reading experience throughout the study assessing its impact on spoken language prediction.

Literate children and adults predict more in spoken language comprehension than nonliterate and low-literate children and adults. An important consequence is that individuals with suboptimal reading behavior, such as people with dyslexia and related reading impairments or healthy readers with low literacy due to infrequent reading practice, are also less proficient oral language comprehenders $[19,95]$. For individuals with dyslexia and related reading disorders, this means that relatively minor impairments that may cause dyslexia can result in greatly exacerbated effects if they result in long-term suboptimal reading experience. Suboptimal reading acquisition and reading practice then results in less sharp representations and hence the formation of fewer and less strong predictive relationships. As a consequence, impaired readers predict less when reading and listening. Healthy individuals with low literacy or illiterate people will suffer detriments similar to those of impaired readers as a consequence of reduced or (in the case of complete illiterates) absent reading experience. Interventions targeted at both healthy low literates and impaired readers thus must at least partly focus on increasing and optimizing the quantitative and qualitative reading experience. Our arguments provide one more reason why more efforts should be undertaken to teach the hundreds of millions of illiterates in developing countries and functional illiterates across the world how to read (or to read better) and why a focus on artificial intelligence voice recognition and voice assistants to overcome literacy-related problems has its dangers.

\section{Box 4. Prediction in Older Adults}

Older adults typically have decades of additional reading experience and often have larger vocabularies than younger adults [110], yet many studies suggest that older adults predict less than younger adults during reading $[111,112]$. There are also studies that suggest that older readers adopt a 'riskier' reading strategy than younger adult readers (older readers skip words more often [113]; cf. [114,115]), consistent with the notion that they rely more on predictions of what the next word will be. One interpretation of such findings is that older adults predict more (or are more likely to act on a prediction) than younger adults to compensate for age-related cognitive decline (in line with evidence that older adults develop strategic differences in reading to overcome age-related differences in processing resources [116]). A recent study investigating prediction in spoken language observed that age was positively related to predictive processing [57]. Interestingly, this effect emerged in the regression analysis only after accounting for age effects on working memory and processing speed (simple correlations suggested a negative relationship between older age and prediction). In other words, older adults' increased lifelong (reading) experience is likely to play a positive role in predictive processing but this may often be offset by age-related cognitive decline in other cognitive abilities such as working memory and processing speed. Future research needs to investigate more systematically the interaction of (reading) experience and cognitive decline on prediction in language processing (ideally in a longitudinal design). 


\section{Acknowledgments}

We thank Michael Cutter, Markus Ostarek, Hugh Rabagliati, and Patrick Sturt for their comments on a previous version of this Opinion article.

\section{References}

1. Morais, J. (2018) Literacy and democracy. Lang. Cogn. Neurosci. 33, 351-372

2. Crain-Thoreson, C. et al. (2001) Parent-child interaction in three conversational contexts: variations in style and strategy. In Sourcebook on Emergent Literacy (Brooks-Gunn, J. and Rebello, P., eds), pp. 23-38, Jossey-Bass

3. Crain-Thoreson, C. and Dale, P.S. (1992) Do early talkers become early readers? Linguistic precocity, preschoo language, and emergent literacy. Dev. Psychol. 28, 412-429

4. Demoulin, C. and Kolinsky, R. (2016) Does learning to read shape verbal working memory? Psychon. Bull. Rev. 23, 703-722

5. Olivers, C.N.L. et al. (2014) The influence of literacy on visual search. Vis. Cogn. 22, 74-101

6. Fernandes, T. et al. (2014) The deficit of letter processing in developmental dyslexia: combining evidence from dyslexics, typical readers and illiterate adults. Dev. Sci. 17, 125-141

7. Carreiras, M. et al. (2009) An anatomical signature for literacy. Nature 461, 983

8. Dehaene, S. et al. (2010) How learning to read changes the cortical networks for vision and language. Science 330, 1359-1364

9. Skeide, M.A. et al. (2017) Learning to read alters corticosubcortical cross-talk in the visual system of illiterates. Sci. Adv. 3, e1602612

10. Altmann, G.T. and Kamide, Y. (1999) Incremental interpretation at verbs: restricting the domain of subsequent reference. Cog nition 73, 247-264

11. Mani, N. and Huettig, F. (2012) Prediction during language processing is a piece of cake - but only for skilled producers. J. Exp. Psychol. Hum. Percept. Perform. 38, 843

12. Staub, A. and Clifton Jr., C. (2006) Syntactic prediction in language comprehension: evidence from either...or. J. Exp. Psychol. Learn. Mem. Cogn. 32, 425-436

13. Van Berkum, J.J. et al. (2005) Anticipating upcoming words in discourse: evidence from ERPs and reading times. J. Exp. Psychol. Learn. Mem. Cogn. 31, 443-467

14. Huettig, F. (2015) Four central questions about prediction in language processing. Brain Res. 1626, 118-135

15. Pickering, M.J. and Gambi, C. (2018) Predicting while comprehending language: a theory and review. Psychol. Bull. 144, 1002-1044

16. Clark, A. (2013) Whatever next? Predictive brains, situated agents, and the future of cognitive science. Behav. Brain Sci. 36, 181-204

17. Friston, K. (2010) The free-energy principle: a unified brain theory? Nat. Rev. Neurosci. 11, 127-138

18. Gibbs, S. (2018) The Guardian. 28 September. https://www theguardian.com/technology/2018/sep/24/speech-recognition-tech-google

19. Mishra, R.K. et al. (2012) Spoken language-mediated anticipatory eye movements are modulated by reading ability: evidence from Indian low and high literates. J. Eye Mov. Res. $5,1-10$

20. Mani, N. and Huettig, F. (2014) Word reading skill predicts anticipation of upcoming spoken language input: a study of children developing proficiency in reading. J. Exp. Child Psychol. 126, 264-279

21. James, A. (2014) Reading Experience Predicts Eye Movements during Online Auditory Comprehension. Published online September 16, 2014. https://www.ideals.illinois.edu/ bitstream/handle/2142/50596/Ariel_James.pdf?sequence $=1$

22. Ng, S. et al. (2018) How struggling adult readers use contextual information when comprehending speech: evidence from event-related potentials. Int. J. Psychophysiol. 125, 1-9

23. Mani, N. et al. (2016) "Pro-active" in many ways: developmental evidence for a dynamic pluralistic approach to prediction. Q. J. Exp. Psychol. 69, 2189-2201
24. Gambi, C. et al. (2018) The development of linguistic prediction: predictions of sound and meaning in 2-to-5 year olds. J. Exp. Child Psychol. 173, 351-370

25. Shipstead, Z. et al. (2012) Is working memory training effective? Psychol. Bull. 138, 628-654

26. Conway, A.R.A. and Getz, S.J. (2010) Cognitive ability: does working memory training enhance intelligence? Curr. Biol. 20, R362-R364

27. Jaeggi, S.M. et al. (2008) Improving fluid intelligence with training on working memory. Proc. Natl. Acad. Sci. U. S. A. 105 6829-6833

28. Buschkuehl, M. et al. (2008) Impact of working memory training on memory performance in old-old adults. Psychol. Aging 23, 743-753

29. Karbach, J. et al. (2015) Adaptive working-memory training benefits reading, but not mathematics in middle childhood. Child Neuropsychol. 21, 285-301

30. Novick, J.M. et al. (2014) Clearing the garden-path: improving sentence processing through cognitive control training. Lang. Cogn. Neurosci. 29, 186-217

31. Payne, B.R. and Stine-Morrow, E.A. (2017) The effects of home-based cognitive training on verbal working memory and language comprehension in older adulthood. Front. Aging Neurosci. 9, 256

32. Bar, M. (2007) The proactive brain: using analogies and associations to generate predictions. Trends Cogn. Sci. 11 280-289

33. Chang, F. et al. (2006) Becoming syntactic. Psychol. Rev. 113, 234-272

34. Chang, F. et al. (2012) Language adaptation and learning: getting explicit about implicit learning. Lang. Linguist. Compass 6 , 259-278

35. Seidenberg, M.S. and McClelland, J.L. (1989) A distributed, developmental model of word recognition and naming. Psychol. Rev. 96, 523

36. Braze, D. et al. (2007) Speaking up for vocabulary: reading skil differences in young adults. J. Learn. Disabil. 40, 226-243

37. Cleland, A.A. and Pickering, M.J. (2006) Do writing and speaking employ the same syntactic representations? J. Mem. Lang. 54, 185-198

38. Ziegler, J.C. and Ferrand, L. (1998) Orthography shapes the perception of speech: the consistency effect in auditory word recognition. Psychon. Bull. Rev. 5, 683-689

39. Phythian-Sence, C. and Wagner, R.K. (2007) Vocabulary acquisition: a primer. Vocabulary Acquisition: Implications for Reading Comprehension, pp. 1-14, Guildford Press

40. Cunningham, A.E. and Stanovich, K.E. (1998) What reading does for the mind. Am. Educ. 22, 8-15

41. Biemiller, A. and Boote, C. (2006) An effective method for building meaning vocabulary in primary grades. J. Educ. Psychol. 98, 44-62

42. Beck, I.L. and McKeown, M.G. (2007) Increasing young lowincome children's oral vocabulary repertoires through rich and focused instruction. Elem. Sch. J. 107, 251-271

43. McKeown, M.G. (1985) The acquisition of word meaning from context by children of high and low ability. Read. Res. Q. 20 , 482-496

44. Anderson, R.C. et al. (1988) Growth in reading and how children spend their time outside of school. Read. Res. Q. 23, 285-303

45. Cain, K. and Oakhill, J. (2011) Matthew effects in young readers: reading comprehension and reading experience aid vocabulary development. J. Learn. Disabil. 44, 431-443

46. Harm, M.W. and Seidenberg, M.S. (1999) Phonology, reading acquisition, and dyslexia: insights from connectionist models. Psychol. Rev. 106, 491-528

47. Seidenberg, M.S. (2005) Connectionist models of word reading. Curr. Dir. Psychol. Sci. 14, 238-242 
48. Andrews, S. (2008) Lexical expertise and reading skill. In The Psychology of Learning and Motivation: Advances in Research and Theory (Vol. 49) (Ross, B.H., ed.), pp. 247-281, Elsevier

49. Perfetti, C.A. (2007) Reading ability: lexical quality to comprehension. Sci. Stud. Read. 11, 357-383

50. Borovsky, A. et al. (2012) Knowing a lot for one's age: vocab ulary skill and not age is associated with anticipatory incremental sentence interpretation in children and adults. J. Exp. Child Psychol. 112, 417-436

51. Nation, K. et al. (2003) Investigating individual differences in children's real-time sentence comprehension using languagemediated eye movements. J. Exp. Child Psychol. 86, 314-329

52. Hintz, F. et al. (2017) Predictors of verb-mediated anticipaton eye movements in the visual world. J. Exp. Psychol. Learn. Mem. Cogn. 43, 1352-1374

53. Rommers, J et al. (2015) Verbal and nonverbal predictors of language-mediated anticipatory eye movements. Atten. Percept. Psycho. 77, 720-730

54. Smalle, E.H.M. et al. (2019) Literacy improves short-term seria recall of spoken verbal but not visuospatial items - evidence from illiterate and literate adults. Cognition 185, 144-150

55. Cowan, N. (2008) What are the differences between long-term, short-term, and working memory? Prog. Brain Res. 169, 323-338

56. MacDonald, M.C. and Christiansen, M.H. (2002) Reassessing working memory: comment on Just and Carpenter (1992) and Waters and Caplan (1996). Psychol. Rev. 109, 35-54

57. Huettig, F. and Janse, E. (2016) Individual differences in working memory and processing speed predict anticipatory spoken anguage processing in the visual world. Lang. Cogn. Neurosci. 31, 80-93

58. Nicolson, R. (1981) The relationship between memory span and processing speed. Intelligence and Learning, pp. 179-183, Springer

59. Bogaerts, L. et al. (2015) Linking memory and language: evidence for a serial-order learning impairment in dyslexia. Res. Dev. Disabil. 43, 106-122

60. Jeffries, S. and Everatt, J. (2004) Working memory: its role in dyslexia and other specific learning difficulties. Dyslexia 10, 196-214

61. Smith-Spark, J.H. and Fisk, J.E. (2007) Working memory functioning in developmental dyslexia. Memory 15, 34-56

62. Huettig, F. et al. (2018) Distinguishing cause from effect - many deficits associated with developmental dyslexia may be a consequence of reduced and suboptimal reading experience. Lang. Cogn. Neurosci. 33, 333-350

63. Silva, C. et al. (2012) Literacy: exploring working memory systems. J. Clin. Exp. Neuropsychol. 34, 369-377

64. Dupoux, E. and Mehler, J. (1990) Monitoring the lexicon with normal and compressed speech: frequency effects and the prelexical code. J. Mem. Lang. 29, 316-335

65. Ziefle, M. (1998) Effects of display resolution on visual performance. Hum. Factors 40, 554-568

66. Rayner, K. et al. (2016) So much to read, so little time: how do we read, and can speed reading help? Psychol. Sci. Public Interest 17, 4-34

67. $\mathrm{Ng}, \mathrm{S}$. et al. (2017) Use of contextual information and prediction by struggling adult readers: evidence from reading times and event-related potentials. Sci. Stud. Read. 21 359-375

68. Payne, B.R. and Federmeier, K.D. (2017) Pace yourself: intraindividual variability in context use revealed by self-paced event-related brain potentials. J. Cogn. Neurosci. 29, 837-854

69. Reichle, E.D. et al. (2003) The EZ Reader model of eyemovement control in reading: comparisons to other models. Behav. Brain Sci. 26, 445-476

70. Rayner, K. (1998) Eye movements in reading and information processing: 20 years of research. Psychol. Bull. 124, 372-422

71. Reichle, E.D. and Laurent, P.A. (2006) Using reinforcement learning to understand the emergence of "intelligent" eyemovement control during reading. Psychol. Rev. 113, 390-408

72. Liu, Y. and Reichle, E. (2010) The emergence of adaptive eye movements in reading. In Proceedings of the Annual Meeting of the Cognitive Science Society (Vol. 32), Cognitive Science Society
73. Marslen-Wilson, W.D. (1987) Functional parallelism in spoken word-recognition. Cognition 25, 71-102

74. Marslen-Wilson, W. and Zwitserlood, P. (1989) Accessing spoken words: the importance of word onsets. J. Exp. Psychol. Hum. Percept. Perform. 15, 576-586

75. Bertram, R. and Hyönä, J. (2003) The length of a complex word modifies the role of morphological structure: evidence from eye movements when reading short and long Finnish compounds. J. Mem. Lang. 48, 615-634

76. Dehaene, S. (2009) Reading in the Brain: The New Science of How We Read, Penguin

77. Shen, W. et al. (2018) The processing of Chinese compound words with ambiguous morphemes in sentence context. $Q$. J. Exp. Psychol. (Hove) 71, 131-139

78. Castles, A. et al. (2018) Ending the reading wars: reading acquisition from novice to expert. Psychol. Sci. Public Interest 19, 5-51

79. Caravolas, M. and Bruck, M. (1993) The effect of oral and written language input on children's phonological awareness: a cross-linguistic study. J. Exp. Child Psychol. 55, 1-30

80. Morais, J. et al. (1979) Does awareness of speech as a sequence of phones arise spontaneously? Cognition 7 , 323-331

81. Morais, J. et al. (1989) Syllabic segmentation and literacy. Lang. Cogn. Process. 4, 57-67

82. Kolinsky, R. and Morais, J. (2018) The worries of wearing literate glasses. Top. Cogn. Psychol. 188, 324-347

83. Baron, J. (2014) The word-superiority effect: perceptual learning from reading. Handb. Learn. Cogn. Process. 6, 131-166

84. Kolinsky, R. et al. (1987) Awareness of words as phonological entities: the role of literacy. Appl. Psycholinguist. 8, 223-232

85. Ramachandra, V. and Karanth, P. (2007) The role of literacy in the conceptualization of words: data from Kannada-speaking children and non-literate adults. Read. Writ. 20, 173-199

86. Ehri, L.C. (2014) Orthographic mapping in the acquisition of sight word reading, spelling memory, and vocabulary learning. Sci. Stud. Read. 18, 5-21

87. Kolinsky, R. (2015) How learning to read influences language and cognition. In The Oxford Handbook of Reading (Pollatsek, A. and Treiman, R., eds), pp. 377-393, Oxford University Press

88. Pattamadilok, C. et al. (2010) How does learning to read affect speech perception? J. Neurosci. 30, 8435-8444

89. Perre, L et al. (2009) Orthographic effects in spoken language: on-line activation or phonological restructuring? Brain Res. $1275,73-80$

90. Ziegler, J.C. and Goswami, U. (2005) Reading acquisition, developmental dyslexia, and skilled reading across languages: psycholinguistic grain size theory. Psychol. Bull. 131, 3-29

91. Araújo, S. et al. (2018) Learning to read facilitates retrieval of phonological representations in rapid automatized naming: evidence from unschooled illiterate, ex-illiterate, and schooled literate adults. Dev. Sci. Published online December 5, 2018. https://doi.org/10.1111/desc.12783

92. Boerma, I.E. et al. (2017) The role of home literacy environment, mentalizing, expressive verbal ability, and print exposure in third and fourth graders' reading comprehension. Sci. Stud. Read. 21, 179-193

93. Mol, S.E. and Bus, A.G. (2011) To read or not to read: a metaanalysis of print exposure from infancy to early adulthood. Psychol. Bull. 137, 267-296

94. Stanovich, K.E. and West, R.F. (1989) Exposure to print and orthographic processing. Read. Res. Q. 24, 402-433

95. Huettig, F. and Brouwer, S. (2015) Delayed anticipatory spoken language processing in adults with dyslexia - evidence from eye-tracking. Dyslexia 21, 97-122

96. Rayner, K. et al. (1983) Latency of sequential eye movements: implications for reading. J. Exp. Psychol. Hum. Percept. Perform. 9, 912-922

97. Reichle, E.D. et al. (1998) Toward a model of eye movement control in reading. Psychol. Rev. 105, 125-157

98. Engbert, R. et al. (2005) SWIFT: a dynamical model of saccade generation during reading. Psychol. Rev. 112, 777-813

99. White, S.J. (2008) Eye movement control during reading: effects of word frequency and orthographic familiarity. J. Exp. Psychol. Hum. Percept. Perform. 34, 205-223 
100. Schotter, E.R. (2018) Reading ahead by hedging our bets on seeing the future: eye tracking and electrophysiology evidence for parafoveal lexical processing and saccadic control by partial word recognition. Psychol. Learn. Motiv. 68, 263-298

101. Reingold, E.M. et al. (2012) Direct lexical control of eye movements in reading: evidence from a survival analysis of fixation durations. Cogn. Psychol. 65, 177-206

102. Cutter, M.G. et al. (2018) Reading sentences of uniform word length - II: very rapid adaptation of the preferred saccade length. Psychon. Bull. Rev. 25, 1435-1440

103. Dikker, S. et al. (2010) Early occipital sensitivity to syntactic category is based on form typicality. Psychol. Sci. 21, 629-634

104. Saenger, P. (1991) The separation of words and the physiology of reading. In Literacy and Orality (Olson, D.R. and Torrance, N., eds), pp. 198-214, Cambridge University Press

105. Sokolov, A.N. (1972) Inner Speech and Thought, Plenum Press

106. Levin, H. and Addis, A.B. (1979) The Eye-Voice Span, MIT Press

107. Fisher, D.F. (1976) Spatial factors in reading research: the case for space. In Eye Movement and Psychological Process (Monty, R.A. and Senders, J.W., eds), Erlbaum Associates

108. Winskel, H. et al. (2009) Eye movements when reading spaced and unspaced Thai and English: a comparison of Thai-English bilinguals and English monolinguals. J. Mem. Lang. 61, 339-351

109. Bai, X. et al. (2008) Reading spaced and unspaced Chinese text: evidence from eye movements. J. Exp. Psychol. Hum Percept. Perform. 34, 1277

110. Verhaeghen, P. (2003) Aging and vocabulary scores: a metaanalysis. Psychol. Aging 18, 332-339
111. Federmeier, K.D. et al. (2010) Age-related and individual differences in the use of prediction during language comprehension. Brain Lang. 115, 149-161

112. Wlotko, E.W. et al. (2012) To predict or not to predict: agerelated differences in the use of sentential context. Psychol. Aging 27, 975-988

113. Rayner, K. et al. (2006) The effect of word frequency, word predictability, and font difficulty on the eye movements of young and older readers. Psychol. Aging 21, 448-465

114. Kliegl, R. et al. (2004) Length, frequency, and predictability effects of words on eye movements in reading. Eur. J. Cogn Psychol. 16, 262-284

115. Laubrock, J. et al. (2006) SWIFT explorations of age differences in eye movements during reading. Neurosci. Biobehav. Rev. $30,872-884$

116. Stine-Morrow, E.A. et al. (2006) Aging and self-regulated language processing. Psychol. Bull. 132, 582-606

117. Johnson, K. (2004) Massive reduction in conversationa American English. In Casual Speech: Data and Analysis. Proceedings of the 1st Session of the 10th International Symposium, Tokyo, Japan (Yoneyama, K. and Maekawa, K., eds), pp. 29-54, National International Institute for Japanese Language

118. Warner, N. (2019) Reduced speech: all is variability. Wiley Interdiscip. Rev. Cogn. Sci. Published online February 27, 2019. https://doi.org/10.1002/wcs.1496

119. Brouwer, S. et al. (2013) Discourse context and the recognition of reduced and canonical spoken words. Appl. Psycholinguist. $34,519-539$ 